\title{
Hydrogen/Deuterium Exchange of Hydrophobic Peptides in Model Membranes by Electrospray Ionization Mass Spectrometry
}

\author{
Raino K. Hansen and R. William Broadhurst \\ Cambridge Centre for Molecular Recognition, Department of Biochemistry, University of Cambridge, Cam- \\ bridge, United Kingdom
}

Paul C. Skelton

Mass Spectrometry Facility, Department of Chemistry, University of Cambridge, Cambridge, United Kingdom

\section{Isaiah T. Arkin}

The Alexander Silberman Institute of Life Sciences, Department of Biological Chemistry, The Hebrew University, Givat-Ram, Jerusalem, Israel

\begin{abstract}
We demonstrate here that the hydrogen/deuterium solvent exchange (HDX) properties of the transmembrane fragment of the M2 protein of Influenza A (M2-TM) incorporated into lipid vesicles or detergent micelles can be studied with straightforward electrospray (ESI) and nanospray mass spectrometry (MS) configurations provided that key factors, including sample preparation techniques, are optimized. Small unilamellar vesicle preparations were obtained by solubilizing dimyristoyl phosphatidylcholine (DMPC) and the M2-TM peptide in aqueous solution with n-octyl- $\beta$-D-glycopyranoside, followed by dialysis to remove the detergent. Electron microscopy experiments revealed that subsequent concentration by centrifugation introduced large multilamellar aggregates that were not compatible with ESI-MS. By contrast, a lyophilization-based concentration procedure, followed by thawing above the liquid crystal transition temperature of the lipid component, maintained the liposome size profile and yielded excellent ion fluxes in both ESI-MS and nano-ESI-MS. Using these methods the global HDX profile of M2-TM in aqueous DMPC vesicles was compared with that in methanol, demonstrating that several amide sites were protected from exchange by the lipid membrane. We also show that hydrophobic peptides can be detected by ESI-MS in the presence of a large molar excess of the detergent Triton X-100. The rate of HDX of M2-TM in Triton X-100 micelles was faster than that in DMPC vesicles but slower than when the peptide had been denatured in methanol. These results indicate that the accessibility of backbone amide sites to the solvent can be profoundly affected by membrane protein structure and dynamics, as well as the properties of model bilayer systems. (J Am Soc Mass Spectrom 2002, 13, 1376-1387) (c) 2002 American Society for Mass Spectrometry
\end{abstract}

S tructural information about integral membrane proteins is scarce, even though they are vital components of many biological systems, such as ion channels. This is in part because they are usually insoluble in water and can become unstable after solubilization with detergents [1]. Furthermore, because they are resistant to structural elucidation by the usual routes of X-ray crystallography and nuclear magnetic resonance (NMR), only $\sim 30$ of the $\sim 10,000$ known protein structures are for membrane proteins, even

Published online October 24, 2002

Address reprint requests to Dr. R. W. Broadhurst, Cambridge Centre for Molecular Recognition, Department of Biochemistry, University of Cambridge, 80 Tennis Court Road, Cambridge CB2 1GA, UK. E-mail: r.w.broadhurst@bioc.cam.ac.uk though they constitute up to $25 \%$ of proteins encoded in the genome sequences of higher organisms [2].

Experimental methods that yield structural details about membrane proteins are therefore highly valuable, including ways of identifying residues that are protected from the solvent, for example by belonging to hydrophobic transmembrane helices or strands. This feat is difficult to demonstrate experimentally, but is necessary for the calibration of algorithms that predict hydrophobic transmembrane (TM) helices from sequence data. One such approach is to follow the kinetics of hydrogen/deuterium solvent exchange (HDX) at backbone amide positions, as exchange rates can be retarded by several orders of magnitude if these sites are protected by hydrogen bonds or steric effects. Several biophysical techniques have been employed to 
monitor amide exchange in membrane proteins, including Fourier transform infrared spectroscopy (FT-IR) [3] and solution [4-6] or solid state [7] NMR. Unfortunately, the information content of these methods is often reduced by poor sensitivity (NMR), lack of sitespecific information (FT-IR), or the need to quench the exchange reaction or manipulate the solvent environment before the extent of exchange can be quantified [4, 6]. HDX has also been studied by mass spectrometry (MS) techniques, with fruitful applications to soluble protein systems in the characterization of tertiary structure, folding, dynamics, and non-covalent interactions $[8,9]$. More recently, attempts have been made to use MS methods for monitoring HDX of membrane proteins in a range of lipid and detergent environments [6, 9-12].

Because detergents bind so tightly to hydrophobic moieties that they are not easily removed, those generally used in the extraction, purification, and reconstitution of membrane proteins have often been regarded as being incompatible with the use of MS [13, 14]. By contrast, soluble proteins do not bind strongly to the ionic detergent sodium dodecyl sulfate (SDS) at concentrations below that required for denaturation [15]. Consequently, soluble proteins can be sequenced in a straightforward manner by MS from a polyacrylamide gel in the presence of SDS [16]. The amphipathic nature of membrane proteins makes them difficult to ionize sufficiently from an aqueous environment in the absence of salt or detergent. MS studies have therefore been carried out mostly with protein fragments generated by either enzymatic or chemical cleavage [17] or by using liquid chromatography (LC) MS with an appropriate organic solvent system in combination with electrospray ionization (ESI) [18, 19]. Current procedures for matrix assisted laser desorption ionization (MALDI) MS permit membrane proteins to be analyzed in the presence of detergent [20]. However, neither MALDI-MS [21, 22] nor organic solvents are ideally suited for real-time studies of HDX kinetics in proteins, as information about the H/D populations of labile sites can be lost when the samples are processed. Successful protocols relying on ESI-MS techniques for membrane proteins solubilized in aqueous detergent or lipid environments have also, until recently, been elusive [10-12, 20].

An ideal HDX experiment would start with a membrane protein reconstituted into an appropriate model system, such as phospholipid vesicles in water. Exchange labeling of labile sites in the protein would then be initiated by dilution into a solvent containing stable isotopes, following which the populations at isotope labeled sites would be quantitated directly, at various time intervals. This would circumvent the need to quench the exchange reaction [6], transfer the sample into a solvent more suitable for measurements of hydrogen/deuterium populations [4] or use proteolytic digestion [23]. A new method introduced by Demmers and co-workers $[10,11]$ meets these requirements by combining HDX of a proteoliposome suspension with nanoelectrospray ionization spectrometry (nano-ESIMS). The work of this group has focused on the interactions between designed $\alpha$-helical transmembrane peptides and large unilamellar vesicles (LUVs) formed by DMPC in water. After initiation of exchange, direct injection of the liposome preparation into the mass spectrometer led to characterization of the global exchange profiles of the hydrophobic peptides, while details of site specific exchange were deduced from collision-induced dissociation tandem mass spectrometry experiments [10]. The results indicate that tryptophan residues are preferentially located at the membrane/water interface and that longer hydrophobic peptides can either adopt a tilted conformation with respect to the membrane normal or distort the bilayer in order to avoid unfavourable interactions with the solvent [11].

For a well characterized membrane protein target, we chose the M2 protein of Influenza $A$, an oligomeric $\alpha$-helical viral ion channel. This small 97 residue integral membrane protein forms proton selective pores which are activated by low $\mathrm{pH}$ environments, such as that found in the endosome after endocytosis of the virus [24, 25]. The M2 channel allows protons to enter the interior of the virus and this acidification causes the matrix protein M1, which coats the viral RNA genome, to dissociate. The replication of Influenza $A$ is inhibited when the M2 channel is blocked by amantadine-related drugs [24], an illustration of the crucial role played by this protein in the survival of the virus [26]. As well as being an important drug target, M2 is a useful minimal model for investigating proton channel proteins. The M2 protein comprises an extracellular N-terminal domain ( $\sim 23$ residues), a TM domain ( $\sim 19$ residues) and an intracellular C-terminal domain ( $\sim 55$ residues) [27]. The active M2 channel is homotetrameric, with monomer units joined together by intermolecular disulphide bridges between cysteine residues that are $\mathrm{N}$-terminal to the TM domain [25]. These covalent links are not required for the function of M2 [28] and tetrameric amantadine-sensitive proton channels can be formed in planar lipid bilayers by a peptide fragment spanning only the TM domain (M2-TM) [25]. Insights from FT-IR [29], solid state NMR [30], and molecular dynamics [31] have yielded a high resolution structure of the monomer backbone of M2-TM and a description of its orientation with respect to the membrane bilayer. These have led in turn to models of the tetrameric M2-TM assembly as a left handed $\alpha$-helical coiled coil containing a central ion-conducting pore through the axis of symmetry. The model structures account for cysteine mutagenesis data, which indicate that Ala-9, Gly-13, His-16, and Trp-20 line the pore of the channel, and are consistent with the imidazole sidechain of His-16 acting as a selectivity filter [32]. In this report we demonstrate that the dynamic properties of the M2-TM peptide incorporated into lipid vesicles or detergent micelles can be studied by HDX with straightforward ESI-MS configurations 
provided that key factors, including sample preparation techniques and standard spectrometer variables, are optimized.

Micellar systems are frequently employed as model membranes because of their small size and ease of use, despite notable differences in curvature, lateral packing pressure and surface charge density effects [33]. ESI-MS has been used in investigations of the non-covalent interactions found between detergent molecules in micelles for some years [34], but few applications to HDX studies of membrane proteins in micelles have been reported. Akashi and Takio [12] recently employed ESI Fourier transform ion cyclotron resonance MS techniques to monitor HDX at amide sites in the amphipathic haemolytic peptide melittin associated with dodecylphosphocholine (DPC) micelles. Here we also describe a simple method for optimizing the detection of ESI-MS signals from the M2-TM peptide in the presence of a large molar excess of the detergent Triton X-100 (TX100), which has often proved difficult to remove once bound to a hydrophobic protein [35]. These results hold promise for wider studies by ESI-MS of the HDX properties of membrane proteins reconstituted into lipid vesicles or detergent micelles.

\section{Experimental}

\section{Materials}

Peptide fragments of the M2 protein of Influenza A were made by solid phase Fmoc synthesis and ordered crude from the HHMI/Keck Facility (Yale University, New Haven, CT). The M2-TM sequence corresponds to the transmembrane domain of the wild-type M2 protein, residues S22-L46 (SSDPL VVAAS IIGIL HLILW ILDRL), with a calculated molecular weight of 2728.3 Da.

Chemicals used were: Acetonitrile, ACN (Riedel-de Häen, Seelze, Germany); $\mathrm{D}_{2} \mathrm{O}$ (Goss Scientific, Essex, UK); dimyristoyl phosphatidylcholine, DMPC (Sigma, Dorset, UK); $d_{1}$-methanol, $\mathrm{CH}_{3} \mathrm{OD}$ (Goss Scientific); $h_{4}$-methanol, $\mathrm{CH}_{3} \mathrm{OH}$ (Riedel-de Häen); n-octyl- $\beta$-Dglycopyranoside, OG (Melford, Suffolk, UK); 2-propanol (Aldrich, Dorset, UK); sodium dihydrogen phosphate (Aldrich); sodium hydroxide (Riedel-de Häen); trifluoroacetic acid, TFA (Acros, Geel, Belgium); trifluoroethanol, TFE (Rathburn, Walkerburn, UK); Triton X-100, TX100 (BDH, Poole, UK); and uranyl acetate (Aldrich). All $\mathrm{H}_{2} \mathrm{O}$ was double distilled (Elgastat, High Wycombe, UK).

\section{Purification of Peptide Samples}

Crude M2-TM peptide was purified by reverse phase HPLC using a method adapted from Kukol et al. [29], applying an isopropanol/acetonitrile gradient in the mobile phase. Briefly, $12 \mathrm{mg}$ of crude peptide was dissolved in $1.8 \mathrm{ml}$ acetonitrile acidified with $0.7 \mathrm{ml}$ TFA, followed by immediate injection onto a $25 \mathrm{ml}$
Jupiter 5C4-300 A column (Phenomenex, Cheshire, UK) that had been equilibrated with buffer A (90\% vol/vol $\mathrm{H}_{2} \mathrm{O}, 6 \% \mathrm{vol} / \mathrm{vol} 2$-propanol, $4 \% \mathrm{vol} / \mathrm{vol}$ acetonitrile, and $1 \%$ vol/vol TFA). Elution was performed using buffer B (57\% vol/vol 2-propanol, 38\% vol/vol acetonitrile, $5 \%$ vol/vol $\mathrm{H}_{2} \mathrm{O}$ and $1 \% \mathrm{vol} / \mathrm{vol} \mathrm{TFA}$ ), using a two-step linear gradient (2 column volumes to $80 \%$ buffer B, followed by 5 column volumes to $95 \%$ buffer B), with the peptide of correct mass emerging at $\sim 84 \%$ buffer B. The pooled fractions were then lyophilized and stored at $-20^{\circ} \mathrm{C}$ until required. The yield of peptide was assessed by dissolving a small quantity in TFE, using a theoretical extinction coefficient for the single tryptophan of $5843 \mathrm{~cm}^{-1} \mathrm{M}^{-1}$ for absorption at $280 \mathrm{~nm}$.

\section{Preparation of Peptide Samples in Lipid Vesicles}

M2-TM was reconstituted into synthetic bilayers as described previously [29] by dissolving $3.5 \mathrm{mg}$ peptide in $1 \mathrm{ml}$ of a solution containing 5\% wt/vol OG [36] and $1.25 \% \mathrm{wt} / \mathrm{vol} \mathrm{DMPC}$, aiming for a lipid/peptide molar ratio of 14 . Solubilization was aided by sonication in a water bath (150 W, Camlab, U.K.) for $16 \mathrm{~min}$, with the temperature rising from $23^{\circ} \mathrm{C}$ to $38^{\circ} \mathrm{C}$. After addition of $1 \mathrm{ml} \mathrm{H} \mathrm{H}_{2} \mathrm{O}$ followed by incubation for $5 \mathrm{~min}$ at $38^{\circ} \mathrm{C}$, the detergent was removed by dialysis against 11 of water in a 12-14 kDa cellulose-ester tube (Spectrum, Laguna Hills, CA) at room temperature for $16 \mathrm{~h}$. This procedure limited the final size of the lipid vesicles, prevented detergent from becoming trapped inside them [35], and also removed impurities remaining from reverse phase HPLC as judged by Q-TOF MS of the sample dissolved in ACN. The final volume of the dialysate was 2.3-3.8 ml. Following this, the DMPC/ M2-TM mixture was equilibrated by dialysis against the amide exchange buffer ( $1 \mathrm{mM}$ sodium phosphate at $\mathrm{pH}$ 7) for $6 \mathrm{~h}$ at $23^{\circ} \mathrm{C}$. The final lipid/peptide molar ratio as judged by amino acid analysis was 50 .

\section{Concentration of Lipid Vesicles by Centrifugation}

After dialysis the vesicles were concentrated by precipitating $2.3 \mathrm{ml}$ of the lipid/protein preparation in a Sorvall centrifuge with a SS-34 rotor (60 min at 50,000 $g$ ). The excess liquid was discarded, leaving the precipitate in $0.7 \mathrm{ml}$. The lipid vesicles were then resuspended by vortex shaking for $1 \mathrm{~min}$.

\section{Concentration of Lipid Vesicles by Lyophilization}

Alternatively, $2.3 \mathrm{ml}$ of dilute lipid vesicle solution was transferred into a plastic tube, frozen in liquid nitrogen, and lyophilized in an Edwards (Eastbourne, UK) Micro Modulyo against a vacuum of 0.1 mbar for $5 \mathrm{~h}$, after which the sample was still moist. Following rapid defrosting to $>24{ }^{\circ} \mathrm{C}$, addition of $\mathrm{H}_{2} \mathrm{O}$ to a final weight of $0.7 \mathrm{~g}$ and vortex shaking for $1 \mathrm{~min}$, a $100 \mu \mathrm{l}$ aliquot was dialyzed in a fixed volume dialysis disc with an 8 
$\mathrm{kDa}$ cut-off (Spectrum) against $1 \mathrm{mM}$ sodium phosphate at $\mathrm{pH} 7.0$ for $6 \mathrm{~h}$.

\section{Electron Microscopy}

Solutions containing lipid vesicles were placed on a thin 300- or 400-mesh copper grid, using adhesion times of $60 \mathrm{~s}$ for dilute samples and $30 \mathrm{~s}$ for concentrated samples. After several washes the samples were stained for $30 \mathrm{~s}$ using a $1 \% \mathrm{wt} / \mathrm{vol}$ solution of uranyl acetate. All images were acquired using a Philips (Surrey, UK) CM100 transmission electron microscope operating at $80 \mathrm{kV}$. Photographs were taken of an area chosen at random for low resolution scans $(3400 \times)$, followed by a high resolution scan $(24,500 \times)$ within the same region. The negatives ( 2 in. $\times 3$ in.) were scanned at 800 d.p.i. and converted into positives. After use in MS experiments, the cone openings of punctured Protana nano-ES capillaries were measured by scanning electron microscopy with a Philips XL30-FEG instrument equipped with an Oxford Instruments cryo-stage operating at 5 $\mathrm{kV}$. As the capillaries already possessed a conductive gold coating, no further preparation was required.

\section{Light Scattering Experiments}

Light scattering experiments on lipid vesicle preparations were performed using a Beckman Coulter (High Wycombe, UK) LS230 Particle Size Analyzer by polarization intensity differential scattering. The sizes and distributions of vesicles in the samples were determined using software supplied by the manufacturer.

\section{Mass Spectrometry of Peptide Samples in Lipid Vesicles}

All samples of M2-TM in DMPC vesicles were mixed with 10 volumes of isotonic dilution buffer prior to data collection on a Micromass (Cheshire, UK) Q-TOF 1 fitted with either an electrospray ionization (ESI) or nano-ESI source. Samples for ESI were injected with a syringe pump (Harvard Apparatus, Kent, UK). Optimal spray of lipid vesicles was obtained at $4 \mu \mathrm{l} / \mathrm{min}$, with the capillary $10 \mathrm{~mm}$ from the cone orifice, the cone and capillary voltages set to 80 and $3000 \mathrm{~V}$, respectively, and the source temperature set to $80^{\circ} \mathrm{C}$. (The cone voltage noted for the Micromass instrument used here is elsewhere referred to as the "skimmer" or "orifice" voltage.) The pressure of nitrogen used for desolvation was set to $1501 \mathrm{~h}^{-1}$. For each spectrum, 10 scans were recorded, using $\sim 100$ pmol of peptide in the sample. With the nano-ESI probe, spray of lipid vesicles was obtained with the cone and capillary voltages set to 140 and $1800 \mathrm{~V}$, respectively, with $2 \mu \mathrm{l}$ of sample loaded into medium gold plated needles (Protana, Odense, Denmark). Small diameter capillary openings (0.5-1.5 $\mu \mathrm{m})$ were prepared by aligning the sealed capillary to within $1 \mathrm{~mm}$ of the cone orifice and inducing breakage by applying a capillary voltage of $1500 \mathrm{~V}$. Large openings $(10-20 \mu \mathrm{m})$ were made by puncturing the capillary by hand with a diamond cutter. For each spectrum 60-180 scans were recorded, using $\sim 10$ pmol of peptide in the sample. Strong signals from M2-TM in DMPC vesicles were also observed on a Micromass Quattro-LC instrument, operating at capillary and cone voltages of 2800 and $80 \mathrm{~V}$, respectively, with the source and desolvation temperatures set to $80^{\circ} \mathrm{C}$.

\section{Mass Spectrometry of Peptide Samples in Methanol}

$2.73 \mathrm{mg}$ M2-TM was mixed with $1 \mathrm{ml} \mathrm{CH}_{3} \mathrm{OH}$ and solubilization was aided by sonication in a water bath (150 W, Camlab) for $16 \mathrm{~min}$. This $1 \mathrm{mM}$ stock solution was used to prepare samples containing $100 \mu \mathrm{M}$ $\mathrm{M} 2-\mathrm{TM}$ in $98 \% \mathrm{vol} / \mathrm{vol} \mathrm{CH}_{3} \mathrm{OH} / 2 \% \mathrm{vol} / \mathrm{vol}$ aqueous sodium phosphate $(1 \mathrm{mM})$ at $\mathrm{pH}^{*} 7$. For studies of HDX, $20 \mu \mathrm{l}$ of this solution was diluted into 10 volumes of either $\mathrm{CH}_{3} \mathrm{OH}$ or $\mathrm{CH}_{3} \mathrm{OD}$ containing $98 \% \mathrm{vol} / \mathrm{vol}$ methanol/2\% aqueous sodium phosphate $(1 \mathrm{mM})$ at $\mathrm{pH}^{*} 7$ and loaded into a Hamilton (Lancashire, UK) glass syringe. Mass spectra were collected on a Micromass Q-TOF 1 fitted with a standard ESI source. Samples were injected with a Harvard syringe pump, employing normal analytical conditions (injection rate $4 \mu \mathrm{l} \mathrm{min}^{-1}$ at $23^{\circ} \mathrm{C}$; capillary voltage $3000 \mathrm{~V}$; cone voltage $35 \mathrm{~V}$; nitrogen drying gas $4001 \mathrm{~h}^{-1}$; capillary $\sim 15 \mathrm{~mm}$ from the cone orifice), with the source temperature reduced to $70^{\circ} \mathrm{C}$. The connecting capillary tube was equilibrated by washing for 5 min with $300 \mu \mathrm{l}$ of either $\mathrm{CH}_{3} \mathrm{OH}$ or $91 \% \mathrm{CH}_{3} \mathrm{OD} / 9 \%$ $\mathrm{CH}_{3} \mathrm{OH}$.

\section{Preparation of Peptide Samples in Detergent Micelles}

A $1 \mathrm{mM}$ solution of M2-TM in TX100 was prepared by mixing $1.5 \mathrm{mg}$ of peptide with $540 \mu \mathrm{l} \mathrm{H}_{2} \mathrm{O}$ containing $1 \% \mathrm{vol} / \mathrm{vol} \mathrm{TX} 100$, followed by sonication in a water bath (150 W, Camlab) for $16 \mathrm{~min}$, with the temperature rising from $23{ }^{\circ} \mathrm{C}$ to $38^{\circ} \mathrm{C}$. This was used as a stock solution for the preparation of samples containing 1-100 $\mu \mathrm{M}$ M2-TM and $0.1-1 \% \mathrm{vol} / \mathrm{vol}$ TX100.

\section{Mass Spectrometry of Peptide Samples in Detergent Micelles}

MS measurements were performed on a Micromass Quattro LC instrument with a Harvard syringe pump pushing an aqueous sample at $2 \mu \mathrm{l} \mathrm{min}{ }^{-1}$ without the assistance of an organic mobile phase. The scanning rate was 12 to $150 \mathrm{Da} \mathrm{s}^{-1}$, depending on the span of $\mathrm{m} / \mathrm{z}$ to be covered. A second series of experiments were performed by loop injection with a 1:1 acetonitrile: $\mathrm{H}_{2} \mathrm{O}$ mobile phase, using a Shimadzu (Duisburg, Germany) LC-9A pump at $4 \mu \mathrm{l} \mathrm{min}^{-1}$. The capillary voltage was 
set to $3200 \mathrm{~V}$ and cone voltages were as stated in the text.

\section{Results and Discussion}

\section{Preparation of Lipid Vesicle Samples}

Several methods are available for producing unilamellar or multilamellar phospholipid vesicles, including: (1) Evaporating a solution of the lipid in an organic solvent to produce a thin film, followed by hydration with an aqueous buffer and shaking, extrusion or irradiation with ultrasound; (2) dissolving the lipid in organic solvent, mixing with aqueous buffer and then removing the solvent by cold distillation under vacuum; (3) adding aqueous buffer to lipid dissolved in a supercritical gas and subsequently reducing the pressure; and (4) preparing lipid-detergent mixed micelles in aqueous buffer, followed by dialysis to reduce the concentration of detergent below its critical limit [37]. Techniques (2) and (3) typically produce liposomes with a broad range of sizes, requiring additional sonication or extrusion steps before small and well defined vesicles are obtained, while (1) and (4) give a more uniform size distribution [37].

The aim of this work was to determine simple methods for studying the backbone amide HDX properties of peptide fragments from membrane proteins incorporated into lipid vesicles or detergent micelles. Amide exchange is often quenched by rapid acidification of the sample to $\mathrm{pH} \mathrm{3,} \mathrm{for} \mathrm{example} \mathrm{prior} \mathrm{to}$ fragmentation by enzymatic cleavage and analysis by MS [38]. We anticipated that initial differences in $\mathrm{pH}$ between the interior and exterior compartments of large multilamellar vesicles could introduce uncertainty into measurements of the exchange time and consequently chose to prepare small unilamellar vesicle (SUV) mixtures. Since the use of organic solvents is often incompatible with retaining native protein structure, we developed a more general approach involving the preparation of aqueous mixed micelles containing the detergent OG and the phospholipid DMPC, followed by the addition of dry lyophilized M2-TM peptide, with mixing facilitated by sonication. In order to obtain SUVs, it was necessary to remove the detergent by rapid dialysis [37]. Transmission electron microscopy demonstrated that this mixed micelle protocol resulted in a homogeneous mixture containing small, spherical unilamellar vesicles, mostly with diameters in the range $25-50 \mathrm{~nm}$, with less than $0.3 \%$ of the sample showing signs of aggregation (Figure 1a). However, the concentration of DMPC in this preparation was only $6 \mathrm{mg}$ $\mathrm{ml}^{-1}$, so after the 11-fold dilution into an isotonic buffer required for HDX experiments, the sample was rather dilute. Preliminary ESI-MS experiments showed that the diluted vesicle mixture was capable of sustaining an ion count of $7900 \pm 300$ per scan over the region of interest $(\mathrm{m} / \mathrm{z} 600-4000)$, with a stable ion flux (Figure 2a). This count gave insufficient sensitivity for ESI-MS
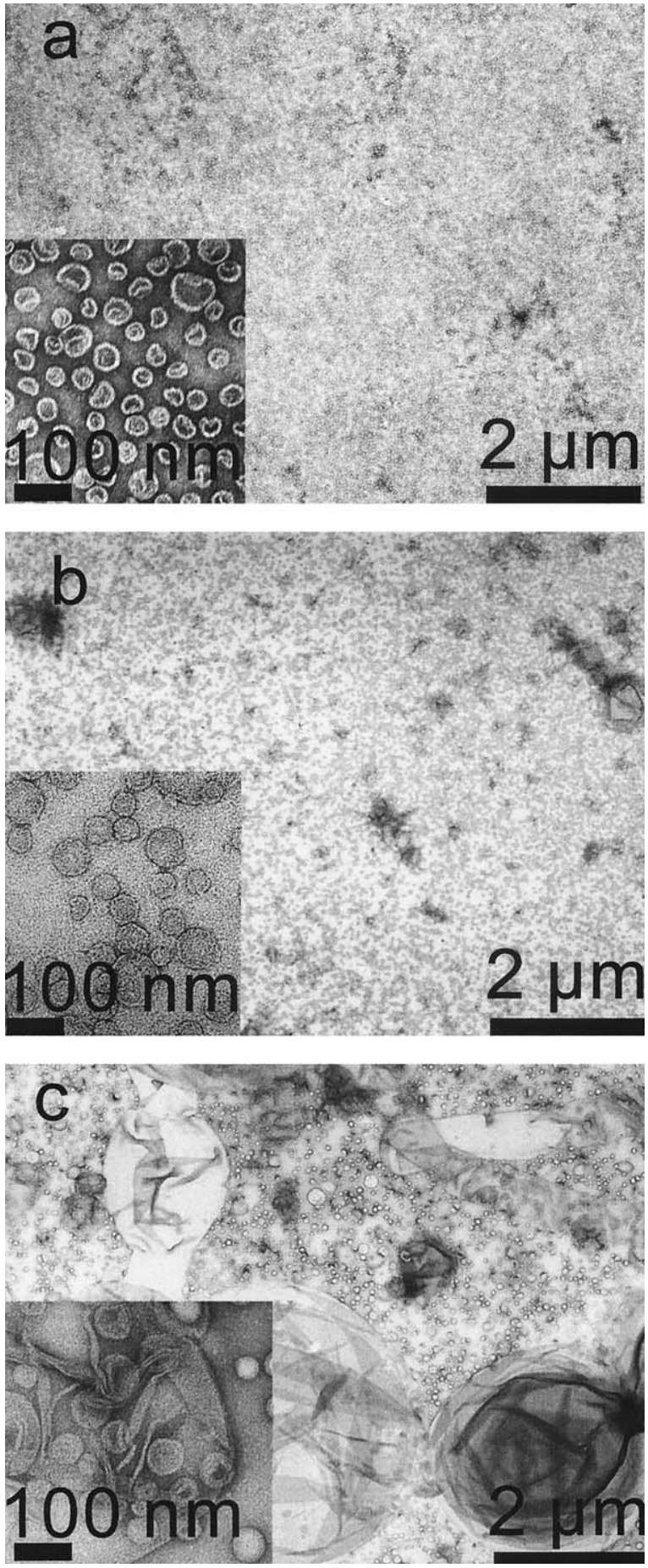

Figure 1. Electron micrographs of M2-TM/DMPC mixtures: (a) Prepared by dialysis of mixed micelles containing protein, lipid, and the detergent OG; (b) concentrated by lyophilization after dialysis; (c) concentrated by centrifugation after dialysis. Experimental methods are detailed in the text.

studies of the M2-TM peptide. We therefore assayed two methods for concentrating the vesicle mixture, centrifugation, and lyophilization, in the hope of obtaining an increased ion count.

Extended centrifugation at 50,000 $\mathrm{g}$ caused the lipid vesicles to precipitate. Excess fluid from the superna- 

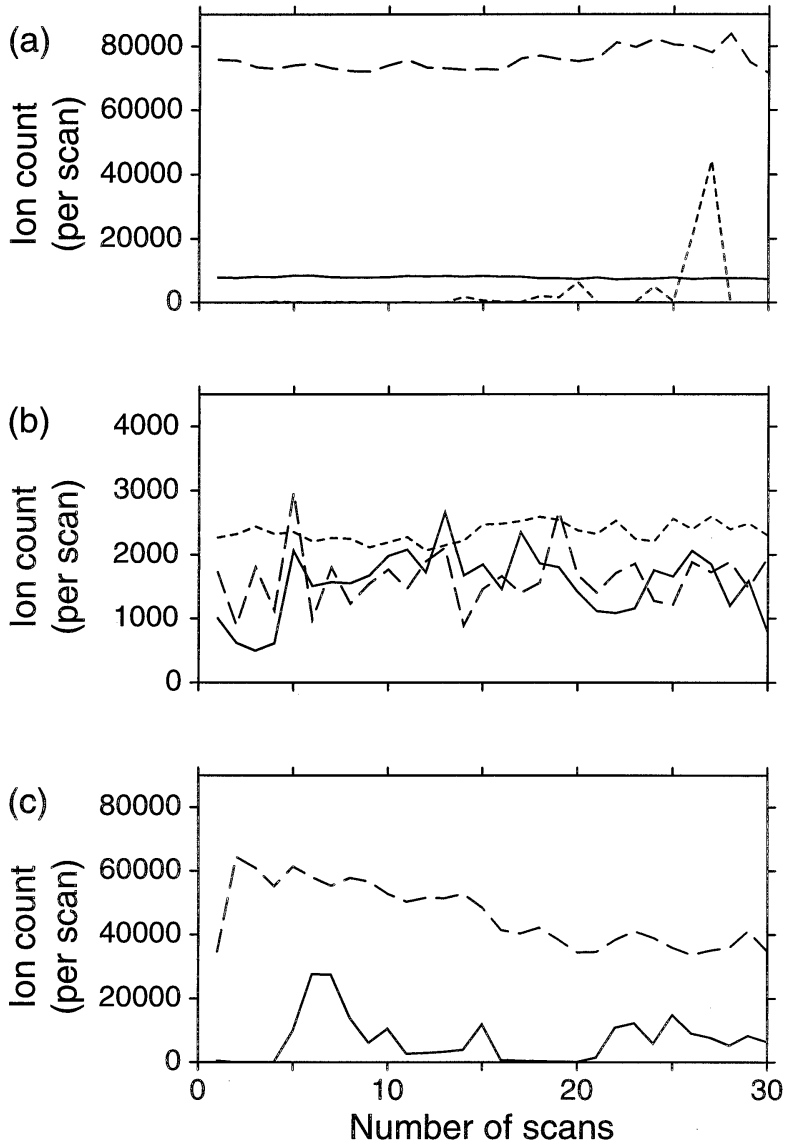

Figure 2. Mass chromatograms over the range 600 to $4000 \mathrm{~m} / \mathrm{z}$ units for M2-TM/DMPC mixtures: Prepared by dialysis of mixed micelles containing protein, lipid, and the detergent OG (unbroken lines); concentrated by centrifugation after dialysis (dotted lines); concentrated by lyophilization after dialysis (dashed lines). (a) ESI-MS using a $100 \mu \mathrm{m}$ diameter steel capillary. (b) NanoESI-MS using capillaries with apertures $10-20 \mu \mathrm{m}$ in diameter. (c) Nano-ESI-MS using capillaries with apertures $0.5-1.5 \mu \mathrm{m}$ in diameter.

tant was discarded, reducing the volume of the sample from 2.3 to $0.7 \mathrm{ml}$, yielding a final lipid concentration of $18 \mathrm{mg} \mathrm{ml}^{-1}$ and a total solid concentration of $21.5 \mathrm{mg}$ $\mathrm{ml}^{-1}$. The vesicles were then resuspended by a short period of vortex shaking. Despite these efforts to concentrate the vesicle mixture, after 11 -fold dilution an even smaller mean ion count of $2700 \pm 8700$ per scan was obtained. Analysis of the chromatogram showed that the flow of ions was highly intermittent (Figure 2a), with occasional intense bursts followed by extended periods during which no ions were detected. Investigation by electron microscopy (Figure 1c) revealed that the centrifugation process had produced a heterogeneous mixture containing a wider range of SUV sizes $(25-75 \mathrm{~nm})$, with many structures larger than $200 \mathrm{~nm}$. Large multilamellar aggregates comprised approximately $64 \%$ of the area of the low resolution EM images. According to light scattering experiments, the mean size of the SUVs present was $65 \pm 8 \mathrm{~nm}$, but a high proportion of the enclosed volume in the preparation was located in a small number of large liposomes of diameter $2 \pm 1 \mu \mathrm{m}$ (data not shown). We concluded that even after dilution, the high degree of aggregation was probably responsible for the poor performance of centrifuged vesicle preparations in the mass spectrometer.

In the lyophilization procedure, a $2.3 \mathrm{ml}$ vesicle sample was frozen in liquid nitrogen and the volume of solvent was reduced by exposure to vacuum for several hours. Aqueous buffer was then added, making the volume up to $0.7 \mathrm{ml}$, followed by vortexing briefly at $30^{\circ} \mathrm{C}$. As shown in Figure 2a, the lyophilized sample behaved well following the 11-fold dilution, giving a tenfold higher mean ion count $(75,900 \pm 3200$ per scan $)$ and maintaining a consistently large ion flux. The electron micrographs displayed in Figure $1 \mathrm{~b}$ indicated that this preparation had retained a larger proportion of SUVs, with most in the size range $50-75 \mathrm{~nm}$. Only $8 \%$ of the area of the low resolution EM images contained multilamellar aggregates, which were also much smaller than the extensive structures observed in the centrifuged preparation. According to light scattering experiments, the mean size of the SUVs was $70 \pm 7 \mathrm{~nm}$ (data not shown). These results indicate that the lower degree of aggregation in the lyophilized sample is much more compatible with electrospray ionization.

Previous studies of the effects of lyophilization have indicated that the size distributions found in lipid vesicle systems are very sensitive to the thawing temperature. Liposomes are often fractured by the formation of ice, but if thawing takes place above the liquid crystal phase transition temperature of the lipid (i.e., $>24^{\circ} \mathrm{C}$ for DMPC), then the membrane is flexible and fractures can reseal, making the retention of SUVs more probable [37]. This explains why small liposomes are retained during the lyophilization procedure employed here. Below the liquid crystal phase transition temperature the membrane is rigid and damage caused by freezing cannot be repaired so easily. Thus, as vesicle fragments slowly diffuse through the sample, exposed sections of the lipid bilayer are likely to meet and fuse, forming large aggregated structures. The increased collision rate resulting from the high local concentration of liposomes produced during centrifugation is also likely to promote the formation of aggregates and large fused vesicles [39].

The method of vesicle preparation employed by Demmers and co-workers for ESI-MS studies of HDX kinetics in transmembrane peptides [10,11] relied on the hydration of thin films of DMPC and peptide deposited by evaporation of a solution in a mixed solvent containing TFE and methanol. This resulted in the formation of extended bilayers, which were converted into LUVs by extrusion through a $400 \mathrm{~nm}$ filter. SUVs can also be prepared by sonicating a dilute solution of DMPC above $24^{\circ} \mathrm{C}$, followed by the removal of large aggregates by centrifugation [40]. However, both of these processes are only capable of producing low concentration proteoliposome preparations. 


\section{Optimizing the Ion Count from Lipid Vesicle Samples}

In our hands, SUV mixtures concentrated by lyophilization yield high, stable ion counts and are much more compatible with ESI-MS than samples concentrated by centrifugation, which contain large multilamellar aggregates (Figure 2a). A droplet of solution will be released from the Taylor cone at the end of a capillary when the charge density on its surface exceeds the surface tension [41]. Once produced, the droplets will decrease in size as a result of solvent evaporation until Coulombic fission occurs, with the same process being repeated in offspring droplets of ever decreasing size [42]. The diameter of the liquid filament emitted from the tip of the Taylor cone is much smaller than the orifice of the capillary [43]. Using equation (6.9) of Cherney [44] and an estimate of the conductivity of an aqueous salt solution [45], we estimate that droplets produced in our ESI-MS experiments (which used steel capillaries with tip openings of $\sim 100 \mu \mathrm{m}$ ) should have a diameter of $\sim 2 \mu \mathrm{m}$, similar in size to the multilamellar aggregates found in the centrifuged preparations. The presence of such large vesicles in the flow of liquid is likely to interfere with the charge circulation pattern at the tip of the Taylor cone and affect the rate of production of charged droplets, with bursts of ions reaching the mass detector only intermittently. The $70 \mathrm{~nm}$ SUVs observed after concentration by lyophilization are probably less disruptive and therefore yield a larger number of charged droplets in a consistent stream.

Coulombic fission is most likely to occur in highly charged droplets, which are formed more readily at low flow rates [43]. Consequently, we employed a flow rate of $4 \mu \mathrm{l} \mathrm{min}{ }^{-1}$ instead of the $10 \mu 1 \mathrm{~min}^{-1}$ rate commonly used in ESI-MS studies of analytes dissolved in organic solvents. We also raised the flow of the desolvation gas to $150 \mathrm{l} \mathrm{h}^{-1}$ in order to increase the rate of evaporation of the less volatile aqueous solvent. The analytical treatment of de Juan and de la Mora [43] suggests that at a flow rate of $4 \mu \mathrm{lmin}^{-1}$ the ratio of the mean charge of the droplets produced by the $100 \mu \mathrm{m}$ capillary to the threshold required for Coulombic fission (i.e., the Rayleigh limit) is $\sim 0.5$. Evaporation processes must therefore reduce the volume of the droplet by approximately two thirds before the first fission can occur. In the case of a droplet containing a single large vesicle surrounded by a layer of water, the solvent would be expected to evaporate until the surface of the vesicle is exposed. The charge density on the surface of this naked liposome may well be below the Rayleigh limit, so if the rate of evaporation is retarded by the lipid bilayer, it is possible that molecular ions will not be produced before the droplet enters the time of flight detector. By contrast, a droplet containing a single SUV should evaporate down to a smaller naked liposome with a much higher surface charge density. Such a particle is much more likely to undergo subsequent fission events resulting in detectable molecular ions.
The extrusion-based preparation method employed by Demmers and co-workers $[10,11]$ should yield LUVs with diameters of several hundred nanometers, slightly smaller than the aggregates observed in our centrifugeconcentrated samples. To implement nano-ESI-MS with their vesicle preparations with good sensitivity, custom-made gold coated borosilicate glass capillary needles with large tip openings $(>10 \mu \mathrm{m})$ were required. We therefore investigated the feasibility of running MS experiments in nanospray mode using standard gold plated glass capillaries with sealed tips fractured in two different ways. Small diameter openings $(0.5-1.5 \mu \mathrm{m}$ determined by scanning electron microscopy) were prepared in the mass spectrometer by using electrical attraction to fracture the sealed capillary. Larger openings (10 $-20 \mu \mathrm{m}$ by electron microscopy) were made by puncturing the capillary by hand with a diamond cutter. As shown in Figure 2b, a stable flux of ions could be achieved in nanospray mode for capillaries with larger openings for all three methods of sample preparation (dialysis, centrifugation, and lyophilization). The mean ion counts obtained (1500-2300 counts per scan) were again too small to be useful for high sensitivity studies of the M2-TM peptide. This differs from the behaviour demonstrated by the small aperture capillaries in Figure 2c: The dilute preparation gave an intermittent ion flux, with a mean count per scan of 6900; no M2-TM ions were detected from centrifuge-concentrated mixtures; and an intense, stable ion flux was achieved for preparations that were concentrated by lyophilization (mean count $45,900 \pm 9800$ per scan).

The only variables that differed between our centrifuged and lyophilized preparations were the size and shape of the lipid vesicles they contained, so these factors must determine the suitability of a particular sample for mass spectrometry. Assuming that the flow rate is proportional to the area of the capillary orifice, we estimate that droplets produced by the larger and smaller aperture nanospray needles have diameters of $\sim 0.4 \mu \mathrm{m}$ and $\sim 0.1 \mu \mathrm{m}$, respectively [44]. The $70 \mathrm{~nm}$ structures that comprise the major fraction of vesicles in the dialyzed and lyophilized preparations are smaller than the jets of droplets emitted by the Taylor cones of both sets of nanospray capillaries. However, Figure $2 b$ shows that these preparations yield low ion counts with 10-20 $\mu \mathrm{m}$ aperture nanospray capillaries. Furthermore, the larger aggregates present in the centrifuged preparation make little difference to the ion flux obtained (Figure 2b). These results imply that an event during the nanospray procedure results in ions from only a tiny fraction of the population of smaller vesicles reaching the detector. Alternatively, since it has long been known that vesicles and lipid coated viruses can fuse under pressure [46], as our own experience with centrifugation has confirmed (see Figure 1c), it may be that the liposomes in all of the preparations undergo a degree of reshaping. Fortunately, for $70 \mathrm{~nm}$ vesicles in the lyophilized preparation the factors responsible for poor performance with larger aperture capillary needles are 

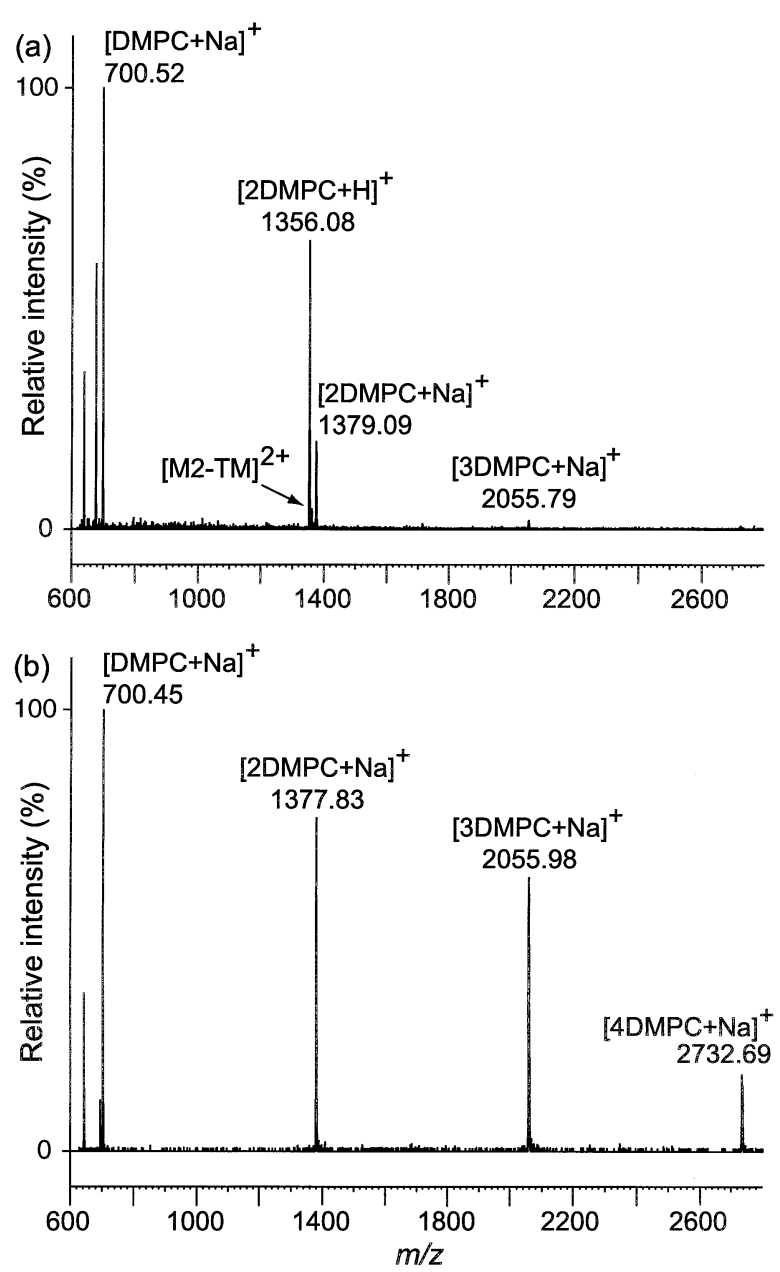

Figure 3. Mass spectra of DMPC vesicle preparations incorporating the M2-TM peptide in protonated aqueous buffer at $\mathrm{pH} 7$ : (a) By ESI-MS; (b) by nano-ESI-MS. Assignments of major quasimolecular ions are indicated.

overcome by reducing the diameter of the orifice to $0.5-1.5 \mu \mathrm{m}$ and a satisfactory ion flux can be obtained.

\section{ESI-MS Spectra of M2-TM in Lipid Vesicles}

Figure 3a displays a typical positive ion ESI mass spectrum of a sample containing the M2-TM peptide reconstituted into vesicles of DMPC, concentrated by lyophilization and then diluted 11-fold into fully protonated aqueous buffer. In agreement with earlier work [47], the most abundant species were monomeric, dimeric, and trimeric ions of DMPC associated with hydrogen and sodium atoms, $\left[n \mathrm{DMPC}+\mathrm{H}^{+}\right.$and $[n \mathrm{DMPC}+\mathrm{Na}]^{+}$, respectively, with protonated ions yielding the most intense signals. The isotope peaks observed within each of these envelopes are separated by $1 \mathrm{~m} / \mathrm{z}$ unit, showing that the quasi-molecular ions were singly charged. The abundance of the DMPC aggregate species decreases rapidly with size, indicating that the lipid vesicles were disrupted efficiently during the ionization process. By contrast, in nano-ESI mass spectra of the same preparation (Figure $3 b$ ) the most abundant lipid species were sodium adducts, and larger clusters were more prevalent. Capillary voltages are usually lower for nano-ESI-MS (e.g., $1800 \mathrm{~V}$ used here compared with $3000 \mathrm{~V}$ for ESI-MS) and this seems to increase the abundance of larger lipid aggregates reaching the detector. When the capillary voltage was decreased to $900 \mathrm{~V}$, a more typical value for nano-ESIMS, the abundance of clustered DMPC species increased further (not shown).

Cations of the M2-TM peptide can also be resolved in the ESI mass spectrum of Figure 3a, but at a much lower intensity. Assuming that neither peptide nor lipid are lost during dialysis, the vesicle preparation should contain a 25-fold molar excess of DMPC over M2-TM. Figure $4 \mathrm{a}$ is an expansion of Figure 3a, highlighting the major M2-TM signal at $\mathrm{m} / \mathrm{z} 1364.9$, which can be compared with the expected mass of $2728.3 \mathrm{Da}$. Whereas Demmers et al. [10] predominantly observed singly charged monomeric sodium adducts of WALP peptides, our procedures yield mostly doubly charged $[\mathrm{M} 2-\mathrm{TM}+2 \mathrm{H}]^{2+}$ ions (with isotope peaks separated by $0.5 \mathrm{~m} / \mathrm{z}$ units) and a smaller population of [M2-TM + $\mathrm{H}+\mathrm{Na}]^{2+}$ species (at $m / z$ 1375.9).

If the proteoliposome preparation is instead diluted into a deuterated aqueous buffer, protons from several sites in the peptide exchange for deuterons within $30 \mathrm{~s}$, shifting the most abundant M2-TM signal to $\mathrm{m} / \mathrm{z} 1371.9$ (Figure $4 b$ ). This result can be interpreted by comparison with ESI-MS spectra of fully protonated M2-TM diluted into $\mathrm{CH}_{3} \mathrm{OH}$ or $\mathrm{CH}_{3} \mathrm{OD}$, displayed in Figure 4c and $\mathrm{d}$, respectively. As expected for a fully protonated environment, the peptide species observed in $\mathrm{CH}_{3} \mathrm{OH}$ (Figure 4c) had $\mathrm{m} / \mathrm{z} 1364.9$, identical to that seen for M2-TM in DMPC vesicles in $\mathrm{H}_{2} \mathrm{O}$ (Figure 4a). $30 \mathrm{~s}$ after dilution into 10 volumes of $\mathrm{CH}_{3} \mathrm{OD}$ at $\mathrm{pH}^{*} 7$ (Figure $4 d$ ), the signal envelope had migrated to a $\mathrm{m} / \mathrm{z}$ value of 1376.9 , already close to the upper boundary of 1381.8 that would correspond to complete exchange at all intrinsically labile sites. This suggests that a significant number of amide sites were being protected from HDX by the membrane when DMPC vesicles that incorporated the M2-TM peptide were diluted into $\mathrm{D}_{2} \mathrm{O}$ (Figure $4 \mathrm{~b})$ but are protected to a lesser extent in methanol (Figure 4d).

\section{ESI-MS Spectra of M2-TM in Detergent Micelles}

Despite recent reports which suggest that the presence of detergent is not compatible with ESI-MS [20], we investigated the possibility of observing signals from the M2-TM peptide solubilized in micelles of TX100. As shown in Figure 5a, when a solution that contained 90 $\mu \mathrm{M}$ M2-TM and $0.1 \%$ TX100 at pH 7.0 was injected directly into the mass spectrometer (i.e., without an organic mobile phase), singly charged cations of TX100 aggregates were readily detected at a standard cone voltage of $30 \mathrm{~V}$, but no peptide signals were observed. However, when the cone voltage was increased to $90 \mathrm{~V}$ a large count appeared at $m / z$ 1365.3, corresponding to 

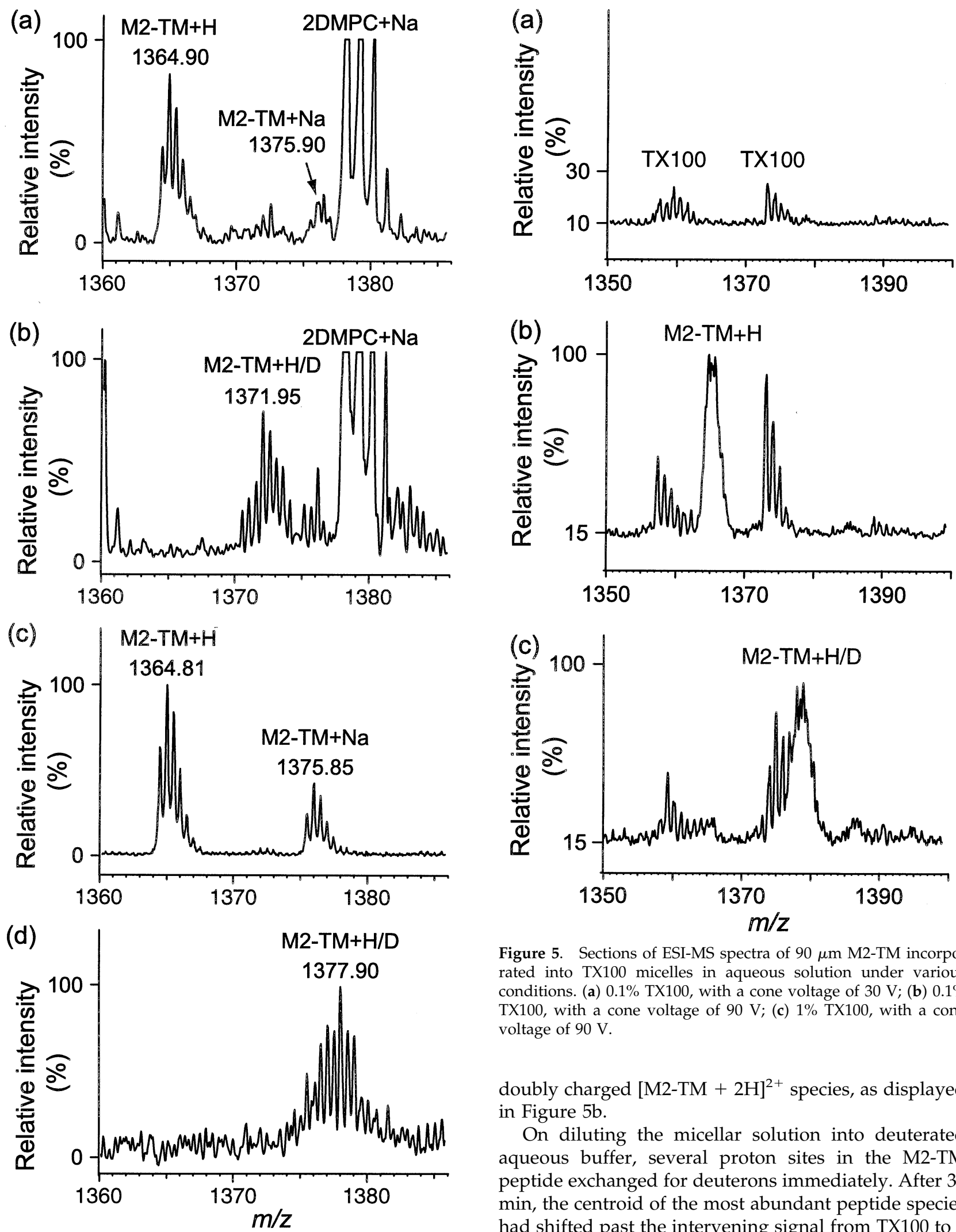

Figure 4. Sections of ESI-MS spectra highlighting isotope envelopes of M2-TM species. (a) M2-TM incorporated into DMPC vesicles in protonated aqueous buffer at $\mathrm{pH}$ 7; (b) M2-TM incorporated into DMPC vesicles, $30 \mathrm{~s}$ after dilution into deuterated aqueous buffer at $\mathrm{pH}^{*}$ 7; (c) M2-TM in fully protonated methanol at $\mathrm{pH} 7$; (d) M2-TM, $30 \mathrm{~s}$ after dilution into deuterated methanol at $\mathrm{pH}^{*} 7$.

Figure 5. Sections of ESI-MS spectra of $90 \mu \mathrm{m}$ M2-TM incorporated into TX100 micelles in aqueous solution under various conditions. (a) $0.1 \%$ TX100, with a cone voltage of $30 \mathrm{~V}$; (b) $0.1 \%$ TX100, with a cone voltage of $90 \mathrm{~V}$; (c) $1 \%$ TX100, with a cone voltage of $90 \mathrm{~V}$.

doubly charged $[\mathrm{M} 2-\mathrm{TM}+2 \mathrm{H}]^{2+}$ species, as displayed in Figure $5 b$.

On diluting the micellar solution into deuterated aqueous buffer, several proton sites in the M2-TM peptide exchanged for deuterons immediately. After 30 min, the centroid of the most abundant peptide species had shifted past the intervening signal from TX100 to a $\mathrm{m} / \mathrm{z}$ value of 1379.4 (Figure $5 \mathrm{c}$ ), close to the boundary indicating complete exchange $(\mathrm{m} / \mathrm{z} 1381.8)$. By way of contrast, the isotope envelope of M2-TM migrated to $\mathrm{m} / \mathrm{z} 1376.1$ after $30 \mathrm{~min}$ of exchange in DMPC vesicles and to $\mathrm{m} / \mathrm{z} 1381.2$ after $30 \mathrm{~min}$ in $\mathrm{CH}_{3} \mathrm{OD}$ (data not 
shown). These results suggest that TX100 micelles are less successful than DMPC vesicles at protecting backbone amide sites in the peptide from exchange with the solvent. The view that TX100 can destabilize native structure in membrane proteins [36] is consistent with the observation that amide exchange in TX100 micelles is nearly as rapid as when M2-TM is denatured in methanol.

Spectra collected over a range of peptide and detergent concentrations suggest that the practical detection limit for M2-TM is quite high for direct injection without an organic mobile phase, at $\sim 10 \mu \mathrm{M}$ in $0.1 \%$ TX100 (data not shown), corresponding to a detergent/peptide molar ratio of 160 . Sensitivity was enhanced dramatically by using a 50\% ACN/50\% water mobile phase, which facilitated the detection of $1 \mu \mathrm{M}$ peptide in $0.1 \%$ TX100, with straightforward discrimination between cations from singly charged detergent and doubly charged peptide species with a cone voltage of $90 \mathrm{~V}$ (data not shown). Unfortunately, these conditions were no longer suitable for HDX experiments, as information about isotope populations was scrambled by the rapid exchange promoted by $\mathrm{ACN}$.

Membrane proteins often go undetected when protein extracts are resolved by two-dimensional polyacrylamide gel electrophoresis, because they are scarcely soluble in the buffers usually used for isoelectric focusing [48]. Attempts to overcome this problem include washing with sodium carbonate or chloroform/ methanol mixtures or sequential extraction with detergents followed by identification using MALDI-MS. Detergents have often been regarded as undesirable contaminants in ESI-MS experiments [13, 14]. For example, a typical protein sequencing protocol incorporates a procedure for removing detergent during the work up for MS [16]. The sample was filtered through pipette tips that contained a bed of reversed-phase silica resin. However, the elution conditions described (60\% methanol in 5\% formic acid) are too mild to ensure recovery of a peptide as hydrophobic as M2-TM, which required a much larger proportion of organic solvent during purification by reverse phase HPLC (see Experimental section). Similarly harsh elution conditions are necessary for many other hydrophobic proteins and peptide fragments [49]. An alternative procedure for removing detergent from peptide/SDS mixtures is by precipitation with cold acetone [14]. We found that M2-TM precipitated along with the detergent when treated in the same way (data not shown) and speculate that this process may substantially reduce the yield of membrane proteins. Glycosylated proteins and hydrophobic peptides created by tryptic digests also bind strongly to detergent (in particular to SDS) and may evade detection for similar reasons. Our experience suggests that in the presence of detergents the detection of membrane proteins by standard ESI-MS methods can be successful if experimental variables (such as the cone voltage) are carefully optimized.

\section{Conclusions}

We have demonstrated that the HDX properties of hydrophobic membrane spanning peptides incorporated into lipid vesicles or detergent micelles can be studied with straightforward ESI-MS and nano-ESI-MS configurations provided that key factors, including sample preparation techniques and standard spectrometer variables, are optimized. Small unilamellar vesicle preparations were obtained by solubilizing DMPC and M2-TM in aqueous solution with the detergent OG, followed by dialysis to remove the detergent. Electron microscopy and light scattering experiments revealed that concentration by centrifugation introduced large multilamellar aggregates, which hindered subsequent studies by ESI-MS. An alternative method of concentration based on lyophilization followed by thawing above the liquid crystal transition temperature was more successful in maintaining the liposome size profile and yielded a high count and a stable flow of ions during electrospray ionization. Low flow rates and increased cone and capillary voltages were employed to increase the production of highly charged droplets that gave rise to mass spectra containing quasi-molecular ions of the peptide amongst more abundant signals from small lipid cluster species. The utility of the method was illustrated by comparing the HDX properties of M2-TM in aqueous DMPC vesicles with those in methanol, demonstrating that several amide sites were protected from exchange by the lipid membrane. In addition, we have optimized routine ESI-MS methods to detect hydrophobic peptides in the presence of a large molar excess of detergent, which is often required for solubilization or maintaining the function of a membrane protein, but can be difficult to remove. The rate of HDX of M2-TM in TX100 micelles was found to be faster than that in DMPC vesicles but slower than when the peptide had been denatured in methanol.

Because HDX rates within a membrane environment can be slow, doubt has been expressed about the ability of such studies to distinguish between folded and unfolded states of integral membrane proteins [33]. However, wide variations in amide exchange timescales have been observed by Fourier-transform infrared spectroscopy studies of intact functional membrane proteins, from minutes for the human erythrocyte glucose transporter [50] and E. coli lac permease 51] to days for the multi-drug transporter EmrE [52]. Together with the difference in global exchange rate profiles described here for M2-TM in vesicle and micellar preparations, these results indicate that the accessibility of backbone amide sites to the solvent can be profoundly affected by membrane protein structure and dynamics, as well as by the properties of model bilayer systems. This work therefore holds promise for wider studies by ESI-MS of the HDX properties of membrane proteins incorporated into lipid vesicles or detergent micelles. 


\section{Acknowledgments}

$\mathrm{RKH}$ is a recipient of a Ph.D. studentship from the Danish Research Training Council and maintenance grants from the Department of Biochemistry, Cambridge; Churchill College, Cambridge; the Lundgren fund, Cambridge; and the Cambridge Philosophical Society. Amino acid analysis was performed by Mr. Peter Sharratt of the PNAC facility at the Department of Biochemistry, Cambridge. The authors are grateful to Professor Nigel Slater and Mr. Richard Taylor for assistance with the particle sizing experiments; to the Multi Imaging Centre, Department of Anatomy, University of Cambridge for EM; and to Professor David Ellar and Dr. Elaine Stimson for stimulating conversations.

\section{References}

1. Bowie, J. U. Stabilizing Membrane Proteins. Curr. Opin. Struct. Biol. 2001, 11, 397-402.

2. Arora, A.; Tamm, L. K. Biophysical Approaches to Membrane Protein Structure Determination. Curr. Opin. Struct. Biol. 2001, 11, 540-547.

3. Rothschild, K. J.; Marti, T.; Sonar, S.; He, Y. W.; Rath, P.; Fischer, W.; Khorana, H. G. Asp(96) Deprotonation and Transmembrane Alpha-Helical Structural-Changes in Bacteriorhodopsin. J. Biol. Chem. 1993, 268, 27046-27052.

4. Dempsey, C. E.; Butler, G. S. Helical Structure and Orientation of Melittin in Dispersed Phospholipid Membranes from Amide Exchange Analysis in Situ. Biochemistry 1992, 31, 11973-11977.

5. Veglia, G.; Zeri, A. C.; Ma, C.; Opella, S. J. Deuterium/ Hydrogen Exchange Factors Measured by Solution Nuclear Magnetic Resonance Spectroscopy as Indicators of the Structure and Topology of Membrane Proteins. Biophys. J. 2002, 82, 2176-2183.

6. Pinheiro, T. J. T.; Cheng, H.; Seeholzer, S. H.; Roder, H. Direct Evidence for the Cooperative Unfolding of Cytochrome $\mathrm{c}$ in Lipid Membranes from H-H-2 Exchange Kinetics. J. Mol. Biol. 2000, 303, 617-626.

7. Cotten, M.; Fu, R.; Cross, T. A. Solid-State NMR and Hydrogen-Deuterium Exchange in a Bilayer-Solubilized Peptide: Structural and Mechanistic Implications. Biophys. J. 1999, 76, 1179-1189.

8. Hernandez, H.; Robinson, C. V. Dynamic Protein Complexes: Insights from Mass Spectrometry. J. Biol. Chem. 2001, 276, 46685-46688.

9. Bouchard, M.; Benjamin, D. R.; Tito, P.; Robinson, C. V.; Dobson, C. M. Solvent Effects on the Conformation of the Transmembrane Peptide Gramicidin A: Insights from Electrospray Ionization Mass Spectrometry. Biophys. J. 2000, 78, 1010-1017.

10. Demmers, J. A. A.; Haverkamp, J.; Heck, A. J. R.; Koeppe, R. E.; Killian, J. A. Electrospray Ionization Mass Spectrometry as a Tool to Analyze Hydrogen/Deuterium Exchange Kinetics of Transmembrane Peptides in Lipid Bilayers. Proc. Natl. Acad. Sci. U.S.A. 2000, 97, 3189-3194.

11. Demmers, J. A. A.; van Duijn, E.; Haverkamp, J.; Greathouse, D. V.; Koeppe, R. E.; Heck, A. J. R.; Killian, J. A. Interfacial Positioning and Stability of Transmembrane Peptides in Lipid Bilayers Studied by Combining Hydrogen/Deuterium Exchange and Mass Spectrometry. J. Biol. Chem. 2001, 276, 34501-34508.

12. Akashi, S.; Takio, K. Structure of Melittin Bound to Phospholipid Micelles Studied Using Hydrogen-Deuterium Exchange and Electrospray Ionization Fourier Transform Ion Cyclotron Resonance Mass Spectrometry. J. Am. Soc. Mass Spectrom. 2001, $12,1247-1253$.
13. Lund, S.; Orlowski, S.; de Foresta, B.; le Champeil, P.; Maire, M.; Møller, J. V. Detergent Structure and Associated Lipid as Determinants in the Stabilization of Solubilized Ca2+-ATPase from Sarcoplasmic Reticulum. J. Biol. Chem. 1989, 264, 49074915.

14. le Maire, M.; Deschamps, S.; Møller, J. V.; Lecaer, J. P.; Rossier, J. Electrospray-Ionization Mass-Spectroscopy on Hydrophobic Peptides Electroeluted from Sodium Dodecyl Sulfate Polyacrylamide Gel Electrophoresis Application to the Topology of the Sarcoplasmic-Reticulum $\mathrm{Ca}^{2+}$ ATPase. Anal. Biochem. 1993, $214,50-57$.

15. Reynolds, J. A.; Tanford, C. The Gross Conformation of Protein-Sodium Dodecyl Sulfate Complexes. J. Biol. Chem. 1970, 245, 5161-5165.

16. Wilm, M.; Shevchenko, A.; Houthaeve, T.; Breit, S.; Schweigerer, L.; Fotsis, T.; Mann, M. Femtomole Sequencing of Proteins from Polyacrylamide Gels by Nano-Electrospray Mass Spectrometry. Nature 1996, 379, 466-469.

17. le Coutre, J.; Whitelegge, J. P.; Gross, A.; Turk, E.; Wright, E. M.; Kaback, H. R.; Faull, K. F. Proteomics on Full-Length Membrane Proteins Using Mass Spectrometry. Biochemistry 2001, 39, 4237-4242.

18. Ball, L. E.; Oatis, J. E.; Dharmasiri, K.; Busman, M.; Wang, J. Y.; Cowden, L. B.; Galijatovic, A.; Chen, N.; Crouch, R. K.; Knapp, D. R. Mass Spectrometric Analysis of Integral Membrane Proteins: Application to Complete Mapping of Bacteriorhodopsins and Rhodopsin. Protein Sci. 1998, 7, 758-764.

19. Whitelegge, J. P.; le Coutre, J.; Lee, J. C.; Engel, C. K.; Prive, G. G.; Faull, K. F.; Kaback, H. R. Toward the Bilayer Proteome, Electrospray Ionization-Mass Spectrometry of Large, Intact Transmembrane Proteins. Proc. Natl. Acad. Sci. U.S.A. 1999, 96 , 10695-10698.

20. le Maire, M.; Champeil, P.; Møller, J. V. Interaction of Membrane Proteins and Lipids with Solubilizing Detergents. Biochim. Biophys. Acta 2000, 1508, 86-111.

21. Figueroa, I. D.; Russell, D. H. Matrix Assisted Laser Desorption Ionization Hydrogen/Deuterium Exchange Studies to Probe Peptide Conformational Changes. J. Am. Soc. Mass Spec. 1999, 10, 719-731.

22. Ghaemmaghami, S.; Fitzgerald, M. C.; Oas, T. G. A Quantitative, High Throughput Screen for Protein Stability. Proc. Natl. Acad. Sci. U.S.A. 2000, 97, 8296-8301.

23. Woods, V. L.; Hamuro, Y. High Resolution, High-Throughput Amide Deuterium Exchange-Mass Spectrometry (DXMS) Determination of Protein Binding Site Structure and Dynamics: Utility in Pharmaceutical Design. J. Cell. Biochem. 2001, 37(Suppl.), 89-98.

24. Hay, A.; Wolstenholme, A.; Skehel, J.; Smith, M. The Molecular Basis of the Specific Anti-Influenza Action of Amantadine. EMBO J. 1985, 4, 3021-3024.

25. Fischer, W. B.; Sansom, M. S. P. Viral Ion Channels: Structure and Function. Biochim. Biophys. Acta. 2002, 1561, 27-43.

26. Takeda, M.; Pekosz, A.; Shuck, K.; Pinto, L. H.; Lamb, R. A. Influenza A Virus M2 Ion Channel Activity is Essential for Efficient Replication in Tissue Culture. J. Virol. 2002, 76, 1391-1399.

27. Hull, J. D.; Gilmore, R.; Lamb, R. A. Integration of a Small Integral Membrane-Protein, M2, of Influenza Virus into the Endoplasmic-Reticulum. Analysis of the Internal Signal-Anchor Domain of a Protein with an Ectoplasmic NH2 Terminus. J. Cell Biol. 1998, 106, 1489-1498.

28. Castrucci, M. R.; Hughes, M.; Calzoletti, L.; Donatelli, I.; Wells, K.; Takada, A.; Kawaoka, Y. The Cysteine Residues of the M2 Protein are not Required for Influenza A Virus Replication. Virology 1997, 238, 128-134.

29. Kukol, A.; Adams, P. D.; Rice, L. M.; Brunger, A. T.; Arkin, I. T. Experimentally Based Orientational Refinement of Membrane 
Protein Models: A Structure for the Influenza A M2 H+ Channel. J. Mol. Biol. 1999, 286, 951-962.

30. Wang, J.; Kim, S.; Kovacs, F.; Cross, T. A. Structure of the Transmembrane Region of the M2 Protein $\mathrm{H}+$ Channel. Protein Sci. 2001, 10, 2241-2250.

31. Forrest, L. R.; Kukol, A.; Arkin, I. T.; Tieleman, D. P.; Sansom, M. S. P. Exploring Models of the Influenza A M2 Channel: MD Simulations in a Phospholipid Bilayer. Biophys. J. 2000, 78, 55-69.

32. Pinto, L. H.; Dieckmann, G. R.; Gandhi, C. S.; Papworth, C. G.; Braman, J.; Shaughnessy, M. A.; Lear, J. D.; Lamb, R. A.; DeGrado, W. F. A Functionally Defined Model for the M2 Proton Channel of Influenza A Virus Suggests a Mechanism for its Ion Selectivity. Proc. Natl. Acad. Sci. U.S.A. 1997, 94, 11301-11306.

33. Booth, P. J.; Templer, R. H.; Meijberg, W.; Allen, S. J.; Curran, A. R.; Lorch, M. In Vitro Studies of Membrane Protein Folding. Crit. Rev. Biochem. Mol. Biol. 2001, 36, 501-603.

34. Siuzdak, G.; Bothner, B. Gas-Phase Micelles. Angew. Chem. Int. Ed. 1995, 34, 2053-2055.

35. Allen, T. M.; Romans, A. Y.; Kercret, H.; Segrest, J. P. Detergent Removal During Membrane Reconstitution. Biochim. Biophys. Acta 1980, 601, 328-342.

36. Gould, R. J.; Ginsberg, B. H.; Spector, A. A. Effects of Octyl $\beta$-Glucoside on Insulin Binding to Solubilized MembraneReceptors. Biochemistry 1981, 20, 6776-6781.

37. Lichtenberg, D.; Barenholz, Y. Liposomes-Preparation, Characterization, and Preservation. Methods Biochem. Anal. 1988, 33, 337-462.

38. Frederiksen, L.; Anton, K.; van Hoogevest, P.; Keller, H. R.; Leuenberger, H. Preparation of Liposomes Encapsulating Water-Soluble Compounds Using Supercritical Carbon Dioxide. J. Pharm. Sci. 1997, 86, 921-928.

39. Wong, M.; Thompson, T. E. Aggregation of Dipalmitoyl Phosphatidylcholine Vesicles. Biochem. 1982, 21, 4133-4139.

40. Grohmann, F. L.; Csempesz, F.; Szogyi, M. Stabilization of Small Unilamellar DMPC-Liposomes by Uncharged Polymers. Colloid Polym. Sci. 1998, 276, 66-71.
41. Taylor, S. G. Disintegration of Water Drops in an Electric Field. Proc. Roy. Soc. A 1964, 280, 383-397.

42. Tang, K.; Gomez, A. On the Structure of an Electrostatic Spray of Monodisperse Droplets. Phys. Fluids 1994, 6, 2317-2332.

43. de Juan, L.; de la Mora, J. F. Charge and Size Distributions of Electrospray Drops. J. Colloid Interf. Sci. 1997, 186, 280-293.

44. Cherney, L. T. Structure of Taylor Cone-Jets: Limit of Low Flow Rates. J. Fluid Mech. 1999, 378, 167-196.

45. de la Mora, J. F.; Loscertales, I. G. The Current Emitted by Highly Conducting Taylor Cones. J. Fluid Mech. 1994, 260, 155-184.

46. Sharp, D. G.; Buckingham, M. J. Electron Microscopic Measure of Virus Particle Dispersions in Suspension. Biochim. Biophys. Acta. 1956, 19, 13-21.

47. Wu, Y.; Wang, J. Z.; Sui, S. F. Characterization of Phospholipids by Electron Impact, Field Desorption, and Liquid Secondary Ion Mass Spectrometry. J. Mass Spectrom. 1997, 32, 616625.

48. Santoni, V.; Molloy, M.; Rabilloud, T. Membrane Proteins and Proteomics: Un amour impossible? Electrophoresis 2000, 21, 1054-1070.

49. Lerro, K. A.; Orlando, R.; Zhang, H. Z.; Usherwood, P. N. R.; Nakanishi, K. Separation of the Sticky Peptides from Membrane Proteins by High Performance Liquid Chromatography in a Normal Phase System. Anal. Biochem. 1993, 215, $38-44$.

50. Alvarez, J.; Lee, D. C.; Baldwin, S. A.; Chapman, D. Fourier Transform Infrared Spectroscopic Study of the Structure and Conformational Changes of the Human Erythrocyte Glucose Transporter. J. Biol. Chem. 1987, 262, 3502-3509.

51. le Coutre, J.; Narasimhan, L. R.; Patel, C. K. N.; Kaback, H. R. The Lipid Bilayer Determines Helical Title Angle and Function in Lactose Permease of Escherichia coli. Proc. Natl. Acad. Sci. U.S.A. 1997, 94, 10167-10171.

52. Arkin, I. T.; Russ, W. P.; Lebendiker, M.; Schuldiner, S. Determining the Secondary Structure and Orientation of EmrE, a Multi-Drug Transporter, Indicates a Transmembrane Four-Helix Bundle. Biochemistry 1996, 35, 7233-7238. 